\title{
BMJ Open Quality SafePsych: improving patient safety by delivering high-impact simulation training on rare and complex scenarios in psychiatry
}

\author{
Kezanne Tong, ${ }^{1}$ Eimear McMahon, ${ }^{2,3}$ Bronwyn Reid-McDermott, ${ }^{2}$ Dara Byrne, ${ }^{4}$ \\ Anne M Doherty (1) ${ }^{5,6}$
}

To cite: Tong K, McMahon E, Reid-McDermott B, et al. SafePsych: improving patient safety by delivering highimpact simulation training on rare and complex scenarios in psychiatry. BMJ Open Quality 2021;10:e001533. doi:10.1136/ bmjoq-2021-001533

- Additional supplemental material is published online only. To view, please visit the journal online (http://dx.doi.org/10. 1136/bmjoq-2021-001533).

Received 19 April 2021 Accepted 27 August 2021

A) Check for updates

(c) Author(s) (or their employer(s)) 2021. Re-use permitted under CC BY-NC. No commercial re-use. See rights and permissions. Published by BMJ.

${ }^{1}$ National Forensic Mental Health Service, Central Mental Hospital, Dundrum, Ireland

${ }^{2}$ School of Medicine, National University of Ireland Galway, University Road, Galway, Ireland ${ }^{3}$ Department of Psychiatry, University Hospital Galway, Galway, Ireland

${ }^{4}$ Irish Centre for Applied Patient Safety and Simulation, National University of Ireland - Galway, Galway, Ireland

${ }^{5}$ Department of Psychiatry, University College Dublin, School of Medicine, Dublin, Ireland

${ }^{6}$ Department of Psychiatry, Mater Misericordiae University Hospital, Dublin, Ireland

Correspondence to Dr Anne M Doherty; anne.doherty@ucd.ie

\section{ABSTRACT}

Introduction Despite an evidence base demonstrating simulation to be an effective medical education tool, it is not commonly used in postgraduate psychiatry training as it is in other medical specialties.

Objective This paper outlines the development and effectiveness of a hybrid-virtual simulation-based workshop designed to improve patient care by improving clinical skills of non-consultant hospital doctors (NCHDs) in detecting and managing rare and complex psychiatric emergencies.

Methods Three clinical vignettes based on near-miss psychiatric cases were developed by a multidisciplinary team of physicians and nurses in psychiatry and experts in simulation-based medical education. The workshop, 'SafePsych' was delivered in a simulation laboratory while and broadcast via Zoom video-conferencing platform to observers. Debriefing followed each clinical scenario. Participants completed preworkshop and postworkshop questionnaires to evaluate clinical knowledge.

Results The workshop was attended by consultants $(\mathrm{n}=12)$ and NCHDs in psychiatry and emergency medicine $(n=19)$, and psychiatric nurses $(n=5)$. In the psychiatry NCHD group, test scores significantly improved following the workshop $(p<0.001)$. There were significant improvements in the test scores with a mean difference of 2.56 (SD 1.58, $p<0.001$ ). Feedback from participants and observers was positive, with constructive appraisals to improve the virtual element of the workshop.

Conclusion Simulation-based training is effective in teaching high risk, rare complex psychiatric cases to psychiatry NCHDs. Further exploration of the learning needs of nursing staff is required. Future workshop delivery is feasible in the COVID-19 environment and beyond, using a virtual element to meet social distancing requirements while enhancing the reach of the training.

\section{INTRODUCTION}

Traditionally, the training of doctors relied on 'learning by doing' approach. Lack of knowledge, experience and clinical supervision among healthcare practitioners is associated with increased patient morbidity and mortality. ${ }^{12}$ While the adage 'see one, do one, teach one' may work for common clinical presentations, it is less applicable to rare cases.
Simulation-based training has developed an evidence base as an effective medical education tool, providing an immersive and experiential learning experience to medical students/postgraduate trainees in a low risk, realistic clinical setting. ${ }^{3-5}$ The use of simulation in medical education is a practical strategy to reduce clinical errors, enhancing patient safety. ${ }^{6-8}$ There is a well-established body of evidence supporting the use of simulation-based training across medical specialties, both in areas of procedure-based skills and in interpersonal-based skills. ${ }^{9-11}$

Psychiatric emergencies frequently occur outof-hours, when a non-consultant hospital doctor (NCHD) 'on-call' is the only psychiatrist physically attending to patients on-site. The ability to recognise and manage these emergencies is key to providing safe, quality psychiatric care, and is limited by lack of experience.

To address this gap, a simulation-based teaching module was developed based on three real-life clinical scenarios that were near-misses in the authors' workplace in the emergency department (ED) and department of psychiatry. This paper outlines the development of a virtual-hybrid simulationbased workshop for rare, complex cases using actors as simulated patients (SP) and a multidisciplinary approach. The aim of the programme was to improve clinical skills of NCHDs in managing rare psychiatric emergencies and reduce risks to patient care by identifying knowledge gaps and areas for improvement in psychiatry trainees. This paper aims to evaluate the effectiveness of this teaching in improving clinicians' knowledge in rare psychiatric cases.

\section{METHODS}

Workshop development

'SafePsych' is a 2.5-hour sustainable and reproducible simulation-based workshop 
developed primarily for psychiatry NCHDs to improve the management of psychiatric and medical emergencies, in collaboration with the Irish Centre for Applied Patient Safety and Simulation (ICAPSS).

Three clinical vignettes were developed by a multidisciplinary team comprising NCHDs, consultants (also the facilitators of the debrief), nurses in psychiatry and experts in simulation-based medical education from ICAPSS. These vignettes were based on three real-life near-miss clinical scenarios. The topics were: neuroleptic malignant syndrome, critically sick patient with anorexia nervosa and management of a psychotic patient presenting with an indictable offence.

Clinical vignettes were shared with SPs in advance of the workshop. SPs were briefed in advance about their role on the day of the workshop by the facilitators, demonstration of the scenario prior to going live.

\section{Recruitment}

Consultants, NCHDs and nurses in the psychiatry department were invited via the departmental email list. From psychiatry, only NCHDs participated in the simulation scenarios as learners. The invitation was extended to emergency medicine staff.

\section{Workshop protocol}

The workshop was delivered in the ICAPSS simulation laboratory. The laboratory cameras broadcast each clinical scenario via Zoom video-conferencing platform to observers unable to enter the simulation laboratory in line with COVID-19 guidelines, in addition to broadcasting to the adjacent debrief room as usual. The SPs, the active learners, simulation technicians and facilitators were present on site.

The workshop was delivered through actors in role of SPs, and members of the mental health and ED teams as confederates (simulated nurse and police officer). Where appropriate, SPs were connected to a vital sign monitoring system, providing live feedback adjusted from the control room. The nurse confederate received prompts from the control room via an earpiece when necessary.

Each scenario lasted approximately $20 \mathrm{~min}$, which included history-taking, clinical investigations and patient management. Workshop observers included consultants and NCHDs in psychiatry and emergency medicine and psychiatric nurses.

\section{Debriefing}

Each clinical scenario was followed by 30 min debriefing facilitated by consultant psychiatrists with expertise in the respective scenarios, using a three-phase debriefing model (reaction, analysis and summary). As part of the debriefing, each participant was encouraged to reflect on their engagement in the scenario. Participants and observers (including those attending remotely) were encouraged to contribute to the discussion. Lastly, the facilitator summarised recommendations for clinical management of the case.

\section{Evaluation}

All learners (NCHDs and nurses) completed preworkshop and postworkshop questionnaires via a web-based survey, comprising 10 clinical multiple-choice questions (MCQs) questions, marked from 10, designed to test knowledge of the clinical scenarios. A feedback survey was distributed to all participants (see online supplemental appendix 1).

\section{Patient and public involvement}

It was not appropriate or possible to involve patients or the public in the design of our research.

\section{RESULTS}

Seventeen NCHDs, 4 nurses and 12 consultants attended from psychiatry. Two NCHDs, and one nurse attended from emergency medicine. The majority of NCHDs $(14 / 19,74 \%)$ completed the preworkshop and postworkshop questionnaire. Following the workshop, mean MCQ scores increased from 4.43 (SD 1.16) to 7.07 (SD 1.64). The mean difference between the two scores was statistically significant $\mathrm{t}(13)=6.84, \mathrm{p}<0.001$.

Among nurses, mean MCQ scores $(4 / 5,80 \%)$ increased from $4.25(\mathrm{SD} 0.96)$ to $6.50(\mathrm{SD} 2.38)$. The difference is not significant, $t(3)=2.03, p=0.135$. When the scores of all learners were combined, mean MCQ scores $(n=18)$ increased from 4.39 (SD1.09) before to 6.94 (SD1.77) after, with statistically significant improvements across all three topics $\mathrm{t}(17)=6.86, \mathrm{p}<0.001$ (table 1 ).

Most (96\%) respondents agreed or strongly agreed that the workshop was useful in addressing training needs and improved their ability to apply their skills. Responders commented that the workshop was more practical than the conventional case-based discussion form of

Table 1 Knowledge-based MCQ scores, before and after SafePsych training on each of the rare specialist topics covered in the training

\begin{tabular}{|c|c|c|c|c|c|c|}
\hline & \multicolumn{3}{|c|}{ Doctors, mean (SD) } & \multicolumn{3}{|c|}{ All participants, mean (SD) } \\
\hline & Before & After & $P$ value/T-value & Before & After & $\mathrm{P}$ value/T-value \\
\hline Total score & $4.4(1.2)$ & $7.1(1.6)$ & $<0.001 / 2.029$ & $4.4(1.1)$ & $7.1(1.8)$ & $<0.001 / 6.862$ \\
\hline Anorexia Nervosa (4MCQs) & $1.6(1.0)$ & $2.4(1.2)$ & $0.008 / 1.192$ & $1.5(1.0)$ & $2.3(1.1)$ & $0.001 / 3.389$ \\
\hline Forensic (3MCQs) & $0.9(0.9)$ & $2.1(0.5)$ & $0.002 / 5.0$ & $0.9(0.8)$ & $2.2(0.5)$ & $<0.001 / 5.047$ \\
\hline
\end{tabular}

MCQ, multiple-choice question. 
postgraduate teaching, especially in the teaching of the legal aspects of psychiatry. A few participants commented on the availability of remote access to NCHDs on rural placements. The main disadvantage noted workshop was the intermittent disruption to digital broadcast.

In the 2 months following the delivery of the workshop, there have been no further instances of two of the clinical scenarios: cases of Neuroleptic Malignant Syndrome or of a psychotic patient presenting following an alleged indictable offence. We have had two presentations of critically sick patients with anorexia nervosa, and on both occasions they were promptly identified and managed in accordance with local guidelines, which suggests that deep learning has been achieved.

\section{DISCUSSION}

There is a need for high-impact training for psychiatry NCHDs in clinical training, particularly in rare and complex cases. The recognition and management of psychiatric emergencies can be challenging, as psychiatry NCHDs are often the 'first responders' to psychiatric emergencies, particularly out-of-hours. Simulation allows clinicians to make errors in low-risk settings, providing the training and experience to manage future emergency situations with live patients. It also offers opportunities to experience rare scenarios, targeting training to NCHDs' needs.

The SafePsych workshop aimed to improve knowledge and clinical skills of NCHDs in managing psychiatric emergencies, and reducing risks to patient care by identifying knowledge gaps and areas for improvement. The significant improvement in the test scores among our workshop participants (especially NCHDs) showed that despite delivery of the workshop using a hybrid model of on-site and virtual learning environment, the quality of the workshop was not compromised. The use of Zoom video-conferencing tool to complement the workshop delivery allowed more attendees from multiple disciplines and specialties to engage in the workshop.

The clinical vignettes represented rare clinical presentations, which require high levels of clinical skill to manage safely, based on near-miss cases in the department. Delayed diagnosis and treatment of neuroleptic malignant syndrome can be fatal, with a mortality rate of up to $10 \%$, and can be difficult to identify. ${ }^{12}$ Anorexia nervosa presenting with dangerously low weight has the highest mortality rates of any psychiatric conditions. ${ }^{13}$ Critically ill patients with anorexia nervosa may need to be treated in a medical setting, but most doctors receive very little training. ${ }^{14}$ Mismanagement of a mentally ill offender may constitute a serious risk to self and others.

Due to the rarity of these cases, it is not always possible for clinicians to be exposed to them in their daily clinical work. Simulation-based training improves clinicians' confidence in managing critical clinical situations,${ }^{15}$ away from patients, with the goal of preventing future harm. ${ }^{7816}$ Structured, facilitated debriefing with reflection after each clinical scenario ${ }^{15}$ is key in addressing the knowledge gap. ${ }^{17}$ The rarity of these cases also presents difficulties in assessing improvements in patients care. Our finding showed improved knowledge and improved patient care in the small number of patients who presented in the 1-month period following the workshop.

Limitations include voluntary attendance: the course may not have reached those who needed it most. Twelve consultant psychiatrists attended the workshop remotely, enabling consultant-led teaching. Organising the workshop was complicated by strict adherence to COVID-19 guidelines. The use of Zoom to enhance attendance increased reach and reduced the training cost per clinician. Another limitation is the use of statistics to evaluate the effect of the intervention on MCQscores. As described in table 1, the changes in MCQ scores are meaningfully improved. However, in capturing qualitative data, we have included meaningful experiential data from the perspective of the learner. ${ }^{18}$

Our evaluation suggested improvement in clinical knowledge, and previous studies demonstrated simulation-based training improves clinician performance and competencies, indirectly improving patient outcome and safety. ${ }^{71619}$ We need to further study participants' knowledge and skills to assess knowledge retention following the workshop, and to study the effect on patient outcomes.

\section{CONCLUSION}

Virtual-hybrid simulation-based psychiatry training is an effective teaching solution to teach rare and complex psychiatric cases, particularly those with high risk, morbidity and mortality. Multidisciplinary development and participation of simulation-based workshop is key to encourage interdisciplinary knowledge sharing. Future workshops should include a virtual element to enhance participation and extend the reach of the training.

Twitter Anne M Doherty @annedohertypsy

Contributors All authors contributed to the development and delivery of the project, its evaluation and the drafting and editing of this manuscript.

Funding This workshop was funded by Spark Seed Funding as part of the Spark Innovation Programme led by the National Doctors and Training Planning (NDTP) Unit, Health Service Executive (HSE).

Competing interests None declared.

Patient consent for publication Not required.

Ethics approval Ethical approval was granted by the Saolta Clinical Research Ethics Committee (Ref CA2060).

Provenance and peer review Not commissioned; externally peer reviewed.

Data availability statement Data are available upon request.

Supplemental material This content has been supplied by the author(s). It has not been vetted by BMJ Publishing Group Limited (BMJ) and may not have been peer-reviewed. Any opinions or recommendations discussed are solely those of the author(s) and are not endorsed by BMJ. BMJ disclaims all liability and responsibility arising from any reliance placed on the content. Where the content includes any translated material, BMJ does not warrant the accuracy and reliability of the translations (including but not limited to local regulations, clinical guidelines, terminology, drug names and drug dosages), and is not responsible for any error and/or omissions arising from translation and adaptation or otherwise. 
Open access This is an open access article distributed in accordance with the Creative Commons Attribution Non Commercial (CC BY-NC 4.0) license, which permits others to distribute, remix, adapt, build upon this work non-commercially, and license their derivative works on different terms, provided the original work is properly cited, appropriate credit is given, any changes made indicated, and the use is non-commercial. See: http://creativecommons.org/licenses/by-nc/4.0/.

ORCID iD

Anne M Doherty http://orcid.org/0000-0002-6995-5361

\section{REFERENCES}

1 Cooper JBet al. Preventable anesthesia mishaps: a study of human factors. Qual Saf Health Care 2002;11:277-82.

2 McQuillan P, Pilkington S, Allan A, et al. Confidential inquiry into quality of care before admission to intensive care. BMJ 1998;316:1853-8.

3 King A, Holder MG, Ahmed RA. Errors as allies: error management training in health professions education. BMJ Qual Saf 2013;22:516-9.

4 Ross AJ, Anderson JE, Kodate N, et al. Simulation training for improving the quality of care for older people: an independent evaluation of an innovative programme for inter-professional education. BMJ Qual Saf 2013;22:495-505.

5 Dave S. Simulation in psychiatric teaching. Adv psychiatr treat 2012;18:292-8.

6 Khan K, Pattison T, Sherwood M. Simulation in medical education. Med Teach 2011;33:1-3.

7 Aggarwal R, Mytton OT, Derbrew M, et al. Training and simulation for patient safety. Qual Saf Health Care 2010;19 Suppl 2:i34-43.

8 Ziv Stephen D Small Paul Root Wolpe A, Paul Root Wolpe A. Patient safety and simulation-based medical education. Med Teach 2000;22:489-95.
9 Legoux C, Gerein R, Boutis K, et al. Retention of critical procedural skills after simulation training: a systematic review. AEM Educ Train 2021;5:e10536.

10 Kaplonyi J, Bowles K-A, Nestel D, et al. Understanding the impact of simulated patients on health care learners' communication skills: a systematic review. Med Educ 2017;51:1209-19.

11 Abdool PS, Nirula L, Bonato S, et al. Simulation in undergraduate psychiatry: exploring the depth of learner engagement. Acad Psychiatry 2017;41:251-61.

12 Ahuja N, Cole AJ. Hyperthermia syndromes in psychiatry. Adv psychiatr. treat 2009;15:181-91.

13 Robinson P, Rhys Jones W, Jones WR. MARSIPAN: management of really sick patients with anorexia nervosa. BJPsych Advances 2018;24:20-32.

14 Ayton A, Ibrahim A. Does UK medical education provide doctors with sufficient skills and knowledge to manage patients with eating disorders safely? Postgrad Med J 2018;94:374-80.

15 Thomson AB, Cross S, Key S, et al. How we developed an emergency psychiatry training course for new residents using principles of high-fidelity simulation. Med Teach 2013;35:797-800.

16 Donaldson L. Safer medical practice: machines, manikins and polo mints. 150 years of the annual report of the chief medical officer on the state of the public health 2008. London: Department of Health, 2009: 49-55.

17 Welke TM, LeBlanc VR, Savoldelli GL, et al. Personalized oral debriefing versus standardized multimedia instruction after patient crisis simulation. Anesth Analg 2009;109:183-9.

18 Price R, Bethune R, Massey L. Problem with $P$ values: why $P$ values do not tell you if your treatment is likely to work. Postgrad Med $J$ 2020;96:1-3.

19 Schmidt E, Goldhaber-Fiebert SN, Ho LA, et al. Simulation exercises as a patient safety strategy: a systematic review. Ann Intern Med 2013;158:426-32. 